\title{
A review of the vehicle suspension system
}

Iyasu JIREGNA, Goftila SIRATA

DOI: 10.30464/jmee.2020.4.2.109

Cite this article as:

Jiregna I., Sirata G. A review of the vehicle suspension system. Journal of Mechanical and Energy Engineering, Vol. 4(44), No. 2, 2020, pp. 109-114.

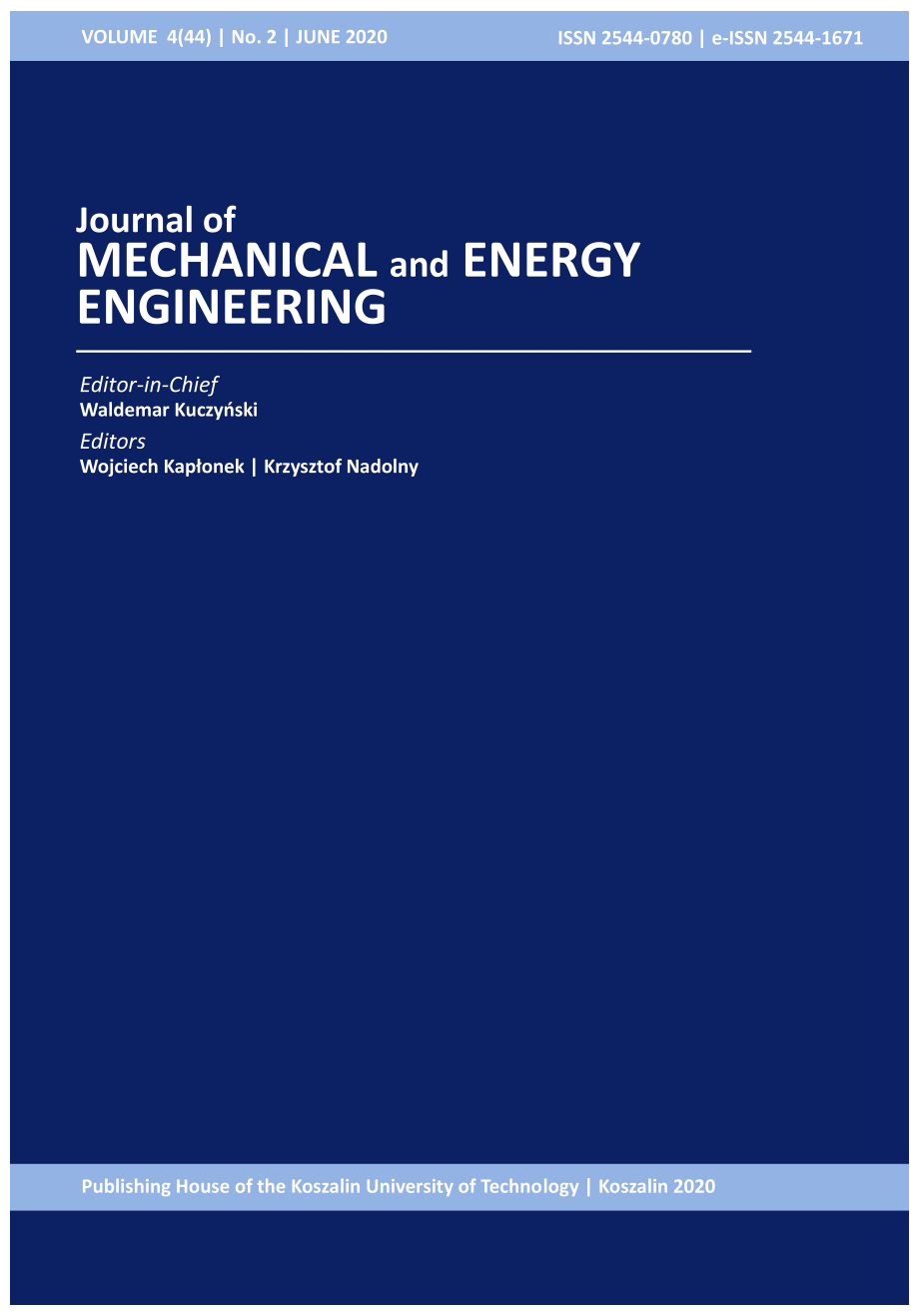

Journal of Mechanical and Energy Engineering

Website: jmee.tu.koszalin.pl

ISSN (Print): 2544-0780

ISSN (Online): 2544-1671

Volume: $4(44)$

Number: 2

Year: 2020

Pages: 109-114

Article Info:

Received 6 April 2020

Accepted 22 May 2020

\section{Open Access}

This article is distributed under the terms of the Creative Commons Attribution 4.0 (CC BY 4.0) International License (http://creativecommons.org/licenses/by/4.0/), which permits unrestricted use, distribution, and reproduction in any medium, provided you give appropriate credit to the original author(s) and the source, provide a link to the Creative Commons license, and indicate if changes were made. 


\title{
A REVIEW OF THE VEHICLE SUSPENSION SYSTEM
}

\author{
Iyasu JIREGNA ${ }^{1 *}$, Goftila SIRATA ${ }^{2}$ \\ ${ }^{1 *}$ Faculty of Mechanical Engineering, Jimma Institute of Techonology, Jimma Ethiopia, \\ e-mail: iyasutafese@gmail.com \\ ${ }^{2}$ Faculty of Material Engineering and Metallurgy, Silesian University of Technology, \\ Krasinskiego 8, 40-019, Katowice, Poland
}

(Received 6 April 2020, Accepted 22 May 2020)

\begin{abstract}
The driving comfort of the vehicle is primarily determined by the design of the suspension system, which transmits the force between the vehicle and the ground. There are different types of vehicle suspension systems, including active suspension systems that provide significant benefits for ride comfort while driving. However, the existing active suspension systems have limited functions such as power, and also complex structure. To overcome the problem, the proper design of the active suspension system by considering its present limitations is essential. A well-designed active suspension system controls the load on the wheels under the resonance of the body structure and ensures driving comfort. It reduces the vibrational energy of the vehicle body caused by the excitation of the road while keeping the stability of the vehicle within an acceptable limit. For a proper design of the active suspension system, the road surface, the seat suspension, and the wheel load are the most important elements to consider. In this study, different types of vehicle suspension systems with their limitations have been thoroughly investigated. Many aspects of control and some of the essential practical considerations are also explored.
\end{abstract}

Keywords: active suspension system, damping coefficient, spring stiffness, vehicle control

\section{INTRODUCTION}

The suspension system is a combination of tires, springs, shock absorbers, and connectors that connect the vehicle to its wheels, allowing the vehicle to travel reasonably well. Suspension systems are essential for both handling and consistency of travel [1]. Currently, the suspension mechanism is one of the most important elements of the car system, which primarily determines the safety of the car and driving comfort. The suspension system transfers and controls the force and reaction (static and dynamic) between the vehicle and the ground [2]. The suspension system is a system of springs, safeguards and associations between the vehicle and its wheels. It is a process that physically separates the car body from the car wheel. The suspension system generally consists of three main components: a structure that maintains the vehicle's weight and determines the suspension geometry; a spring that transforms kinematic energy into potential energy; or vice versa; and a shock absorber, a mechanical device designed to dissipate kinetic energy [3]. Shock absorbers are hydraulic pump type devices that help manage the impact and recoil movement of the vehicle's springs and suspension. The primary role of the shock absorber is to ensure that the vehicle tire remains in contact with the road surface at all times, resulting in the safest control and braking response of the vehicle. The Figure 1 shows an example of the shock absorber, which used in the vehicle suspension system.

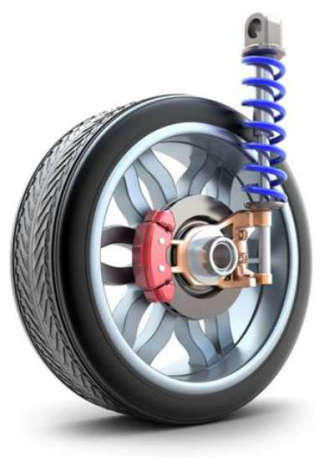

Fig. 1. An illustration of a shock absorber used in automobiles [4] 
The predominant role of the vehicle suspension system is to reduce the vertical acceleration that is transmitted to the vehicle body (indirectly to passengers or loads), ensuring comfort directly in the lane. Therefore, The purposes of the suspension system are: to prevent the road shocks from being transmitted to the components of the vehicle; to protect the resider from road shocks; to maintain the vehicle's stability during pitching or rolling while the vehicles are in motion [4].

The increasing demands on the overall dynamics and stability of vehicles, as well as the rapid development of hybrid and electric passenger cars, could contribute to the implementation of several controlled suspension systems and their integration into the overall control of passenger vehicles [1]. Several scientists have paid great attention to car suspension system like how to ensure the stability of suspension systems and how to improve the required suspension performance, namely driving comfort, road handling, and suspension deflection [4, 5]. Until now, many vehicle suspensions models have been proposed. Modifying the suspension requires a deep understanding of car suspension networks and vehicle dynamics. Keeping the wheel in contact with the road is essential for the safety of the vehicle movement, since the total load of the car is lies through the contact surfaces of the tires [6-8].

\section{CLASSIFICATIONS OF AUTOMOTIVE SUSPENSIONS SYSTEMS}

There are three sorts of vehicle suspension frameworks namely; active, semi-active, and passive suspension frameworks that various specialists have created utilizing various methods and algorithms. Contrasted with the semi-active and active suspension framework, the passive suspension framework has no vehicle steadiness [9]. The dynamic behavior of passive automotive suspension systems is determined by choosing the appropriate spring stiffness (due to the spring type and it characteristic) and shock absorbers characteristic represented by the damping coefficient $[10,11]$.

\subsection{Passive suspensions system}

The passive suspension system shown in Figure 2 is suspension setups comprising of viscous linear springs and dampers with a consistent stiffness and damping coefficient, respectively [12]. The passive suspension of the vehicle is reliable, simple, and economical. With this device, the shock absorber and spring are clamped between the body frame and the wheel bracket. A rod is used to push the piston from the outside. Piston movement is possible through holes, through which liquid can flow through the cylinder sections. This liquid flow produces a reaction forces relative to the speed flow which depends on proportional between displacement of un-sprung and sprung masses.

Damping is achieved by converting the energy of the oscillations into heat and its dispersion to the atmospheric air. This system cannot provide adequate results to solve suspension problems because it does not provide external control and significant changes in materials, valves, or even shapes [13]. The fixed damper and spring section of the passive system is not good enough to absorb energy to withstand the load or road disturbance that has occurred in the vehicle system. The mathematical description of equation of motion for passive suspension system shown in Figure 1, can be given as illustrated in Equations 1 and 2:

$$
\begin{gathered}
M_{S} \ddot{z}_{S}=K_{S}\left(z_{U}-z_{S}\right)+C_{S}\left(\dot{z}_{U}-\dot{z}_{S}\right) \\
M_{U} \ddot{z}_{U}=K_{t}\left(z_{O}-z_{U}\right)-K_{S}\left(z_{U}-z_{S}\right)-C_{S}\left(\dot{z}_{U}-\dot{z}_{S}\right)
\end{gathered}
$$

where: $z_{S}$-vertical displacement of the sprung mass $[\mathrm{m}]$, $z_{U^{-}}$-vertical displacement of the unsprung mass $[\mathrm{m}], z_{O^{-}}$ road unevenness input $[\mathrm{m}], M_{S^{-}}$sprung mass $[\mathrm{kg}], M_{U^{-}}$ unsprung mass $[\mathrm{kg}], K_{S}$-spring stiffness $[\mathrm{kN} / \mathrm{m}], K_{t}$-tire stiffness $[\mathrm{kN} / \mathrm{m}], C_{S^{-}}$-damping coefficient $[\mathrm{kN} . \mathrm{s} / \mathrm{m}]$

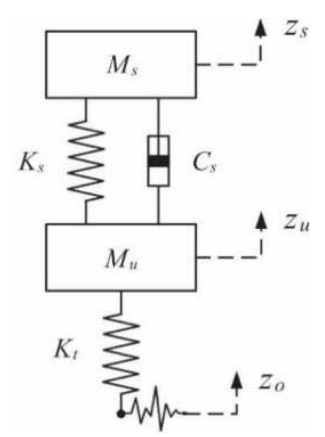

Fig. 2. A theoretical model of passive suspension [14]

\subsection{Semi-active suspension system}

The semi-active suspension system utilizes a variable damper or other variable dispersal parts. One example of a variable dissipater is a viscous twin-tube damper, where the damping coefficient can be changed by changing the hole diameter in the piston. A rheological magnet (MR) damper is an example of semi-active dissipator, which uses rheological magnet fluid. MR fluids are materials that react upon an applied magnetic field with a shift in rheological behavior. This change is usually reflected in the advancement of yield stress which increases the dissipative power of the damper by controlling the electromagnetic field with the magnetic field applied. Semi-active suspension systems have been studied in several ways to minimize actuation energy consumption. The semi-active suspension is like a passive suspension with just a distinction in having a variable damping coefficient but additionally a fixed spring consistent and furthermore a lack of active power sources. This type of device allows smooth switching between passive dampers with a coefficient of semi-active damping, as shown in Figure 3.

The Equation of motion for semi-active suspension system for the case of two degrees of freedom can be given as: 


$$
\begin{gathered}
m_{s} \ddot{x}_{s}+k_{s 1}\left(x_{s}-x_{u}\right)+k_{s 2}\left(x_{s}-x_{u}\right)^{2} \\
+k_{s 3}\left(x_{s}-x_{u}\right)^{3}+C_{s 1}\left(\dot{x}_{s}-\dot{x}_{u}\right) \\
+C_{s 2}\left(\dot{x}_{s}-\dot{x}_{u}\right)^{2}+f_{d}=0, \\
m_{u} \ddot{x}_{u}+k_{t 1}\left(x_{u}-x_{t}\right)+k_{t 2}\left(x_{u}-x_{t}\right)^{2}+k_{t 3}\left(x_{u}-x_{t}\right)^{3} \\
-k_{s 1}\left(x_{s}-x_{u}\right)+k_{s 2}\left(x_{s}-x_{u}\right)^{2}-k_{s 3}\left(x_{s}-x_{u}\right)^{3} \\
-C_{s 1}\left(\dot{x}_{s}-\dot{x}_{u}\right)-C_{s 2}\left(\dot{x}_{s}-\dot{x}_{u}\right)^{2}-f_{d}=0,
\end{gathered}
$$

where: $M_{s}$-sprung mass, $M_{u}$-unsprung mass, $K_{s}$-spring stiffness involving $k_{s 1}, k_{s 2}$ and $k_{s 3}$ as suspension stiffness coefficients and $k_{t l}, k_{t 2}$ and $k_{t 3}$ as tire stiffness coefficients, $\mathrm{C}_{\mathrm{s}}$-suspension damper involving $C_{s I}$ and $C_{s 2}$ are suspension damping coefficients, $f_{m r}$-the controllable damping force generated by MR damper, $K_{t}$-the tire stiffness, $f_{d}$-nonlinear damping force.

The equations 3 and 4 are found using Newton's second law and these are non-linear equations. Because tire stiffness is expressed in the polynomial function of third-order whereas, the damper is expressed in the polynomial function of second-order Sawant and Tamboli [15].

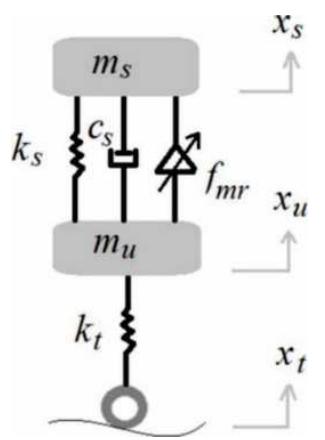

Fig. 3. A theoretical model of a semi-active suspension system [16]

While maintaining a fixed use of the spring, the continuously variable damper modifies the workability in real-time through designing the closed-loop feedback control to ensure adequate energy dissipation, improving the overall efficiency of the suspension compared to the passive system [17]. A semi-active suspension can be electrically controlled remotely to harden or soften the mechanism so that the damping coefficient varies continuously or discontinuously. Switching to work techniques such as raising the suspension helps vehicles turn, brake, accelerate, reduce the low-frequency response to the inertial forces related to rolling and pitching, and prevent resonance of wheels, and excitement of the body.

\subsection{Active suspension system}

Active suspensions are outfitted with electronic control frameworks that control the activity of the suspension components. The example of active suspension, as shown in Figure 4, consists of the actuator, the mechanical spring and the shock absorber. They do not have a limited performance like passive suspensions and build a new advance to eliminate the difficulty of the designing a compromise present in passive suspensions. The actuator in active suspension systems allows the suspension to absorb energy of acceleration of the wheel, and therefore, minimize the acceleration of the vehicle body.

Such systems have much more sensitive capabilities to induced vertical forces caused by unpredictable changes in road access, as the actuator force controls both shock absorbers. This actuator works by having or distributing system power and can be moderated by different types of controllers depending on the supplied design. Active suspension can result in a better balance between vehicle handling comfort and driving stability, resulting in an improved suspension design with proper control methods.

The mathematical description of equation of motion for active suspension system shown in Figure 3, can be obtained from Newton's law of motion as shown in Equations 1 and 2). In this law the total force (F) applied on the unsprung and sprung masses is equal with the product of their masses and acceleration. Thus, the mathematical expression of sprung and unsprung active suspension system can be expressed as follows [18].

For the case of sprung mass:

$$
\begin{gathered}
m_{s} \ddot{z}_{s}+F_{d}\left(\dot{z}_{s}-\dot{z}_{u}\right)+F_{s}\left(z_{s}-z_{u}\right)=0, \\
\Sigma F m_{s}=F_{d}\left(\dot{z}_{u}-\dot{z}_{s}\right)+F_{s}\left(z_{u}-z_{s}\right), \\
\Sigma F m_{s}=m_{s} \ddot{z}_{s} .
\end{gathered}
$$

For the case of unsprung mass:

$$
\begin{gathered}
m_{u} \ddot{z}_{u}-F_{d}\left(\dot{z}_{s}-\dot{z}_{u}\right)-F_{s}\left(z_{s}-z_{u}\right) \\
+F_{t}\left(z_{u}-z_{r}\right)=0 \\
\Sigma F m_{s}=F_{d}\left(\dot{z}_{s}-\dot{z}_{u}\right)+F_{s}\left(z_{s}-z_{u}\right)-F_{t}\left(z_{u}-z_{r}\right) \\
\Sigma F m_{s}=m_{u} \ddot{z}_{u},
\end{gathered}
$$

where: $z_{S}$-vertical displacement of the sprung mass $(\mathrm{m})$, $z_{U}$-vertical displacement of the unsprung mass $(\mathrm{m}), z_{r^{-}}$ vertical displacement due to road input (m), $M_{S}$-sprung mass $(\mathrm{kg}), M_{U}$-unsprung mass $(\mathrm{kg}), F_{S}$-spring stiffness $(\mathrm{kN} / \mathrm{m}), F_{t}$-tire stiffness $(\mathrm{kN} / \mathrm{m}), F_{d}$-damping coefficient between sprung and unsprung masses $(\mathrm{kN} . \mathrm{s} / \mathrm{m}), F_{b^{-}}$ Damping coefficient between road and unsprung mass (kN.s/m)

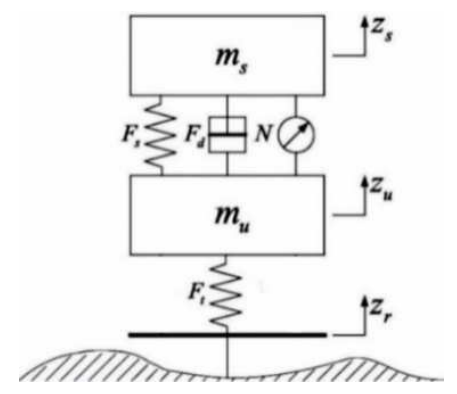

Fig. 4. A model of active suspension system [19] 
Several researchers have recently invested in active suspension systems to improve the stability and driving capabilities of the vehicles. To date, numerous control approaches have been utilized in the active suspension system fields, for example, Quadratic Linear Control (LQR), Quadratic Gaussian Linear Control (LQG), Slider Mode Control, Control H, Adaptive Slider, Preview Control, Neural Network Methods, Fuzzy Logic, and Optimal Control. Control methods can help improve the performance of the active suspension system.

As a result, most researchers have recognized this type of active suspension system is one of the best ways to improve overall performance due to its ability to manage different parameters [17]. Yet it is a complicated and expensive method that justifies a small number of high-quality passenger car or truck models for this specific use of the system [13].

\subsection{Hydraulic or pneumatic active suspensions}

The active suspension system with hydraulic or pneumatic actuator is operated by means of electrical drives, and power is supplied from either a battery source or from conventional ICE itself. Due to their simplicity of design, high strength density, sophisticated technology, reliability, as well as accessibility of various commercial parts, active suspension with hydraulic systems is widely used in body control systems. For instance, a Citroen pneumo-hydraulic suspension (hydroactive system 1st and 2nd generation) has late built up an anti-roll operating system by adjusting the rotary hydraulic actuator at the rear of the vehicle's anti - roll bar center. Another example was the Mercedes Active Body Control System, which uses high-pressure hydraulics to pre-stress the springs and thus produces anti-rolling forces without the right and left wheels being coupled [20]. However, the use of a hydraulic system has drawbacks, such as:

- inefficiently performed because a pressurized system is continuously required,

- relatively high system time constant (flexible hoses and loss of pressure),

- environmental damage due to the presence of toxic hydraulic fluids caused by leaks and rupture of the hose,

- system volume and infeasibility requirements, even if they contribute primarily to the overall jump mass.

\subsection{Electromagnetic active suspensions}

The electromagnetic active suspension system consists of a spring actuator and an electromagnetic actuator set up in parallel configurations within a sprung and unsprung mass. The electromagnetic actuator performs the control systems implemented concerning the electrical range supplied. It produces activecontrolled forces to absorb road shocks quickly, suppress pitch and roll movements, and improve comfort and safety. Bi-directional electromagnetic actuator, on the other hand, works by producing and reducing electrical power consumption compared to the hydraulic actuator [12].
The main superiority of the electromagnetic actuators after comparison with the hydraulic actuators is shown in Table 1.

Tab. 1. Advantages of Electromagnetic actuater over Hydraulic actuater [21]

\begin{tabular}{|c|c|c|}
\hline Characteristics & Hydraulic & Electromagnetic \\
\hline Control & User must & $\begin{array}{l}\text { Motion control } \\
\text { capability with } \\
\text { electronic controller. }\end{array}$ \\
\hline Position accuracy & $\begin{array}{l}\text { Mid-stroke } \\
\text { positioning } \\
\text { requires } \\
\text { Additional } \\
\text { components } \\
\text { and user } \\
\text { support }\end{array}$ & $\begin{array}{l}\text { Positioning capabilities } \\
\text { and velocity control } \\
\text { Allow for } \\
\text { synchronization. }\end{array}$ \\
\hline Environmental & May leak & Minimal \\
\hline Efficiency & Low & High \\
\hline Utilities & $\begin{array}{l}\text { Pump, Power, } \\
\text { Pipes }\end{array}$ & Only power \\
\hline Maintenance & Complex & Simple \\
\hline Operating cost & High & Low \\
\hline Maintenance cost & High & Low \\
\hline
\end{tabular}

Whereas the drawbacks of electromagnetic actuater with respect to hydraulic actuater are Table 2 .

Tab. 2. Drawbacks of electromagnetic actuater [21]

\begin{tabular}{lll}
\hline Characteristics & Hydraulic & Electromagnetic \\
\hline Complexity & $\begin{array}{l}\text { Moderately } \\
\text { complex system } \\
\text { composition }\end{array}$ & $\begin{array}{l}\text { Control system and } \\
\text { motion component } \\
\text { can work together } \\
\text { in multiple } \\
\text { complex } \\
\text { configuration }\end{array}$ \\
\hline Peak power & Very high & High \\
\hline Load rating & Extremely high & $\begin{array}{l}\text { Can be high } \\
\text { depending on the } \\
\text { speed and } \\
\text { load rating }\end{array}$ \\
\hline Acceleration & Very high & Moderate \\
\hline
\end{tabular}

\section{SUMMARY OF ANALYSIS OF VEHICLE SUSPENSION SYSTEMS}

Different types of vehicle suspension systems have been analyzed and summarized as shown in Table 3 . Based on this analysis, the active suspension system with electromagnetic actuators is better than the hydraulic one. There are certain limitations of the active electromagnetic suspension system in terms of complexity and structure. However, it is more efficient as described in Table 1. These limitations still need to be improved. Table 3 gives an overview of the different suspension systems with their limits. 
Tab. 3. Suspension systems with their limits.

\begin{tabular}{lllll}
\hline \multirow{2}{*}{ Parameters } & \multirow{2}{*}{$\begin{array}{l}\text { Passive } \\
\text { suspensions }\end{array}$} & $\begin{array}{l}\text { Semi-active } \\
\text { suspensions }\end{array}$ & \multicolumn{2}{l}{ Active suspension systems } \\
\cline { 4 - 5 } Structure & Simplest & Complex & Most complex & Simple \\
\hline Weight / Volume & Lowest & Low & High & Highest \\
\hline Cost & Lowest & Low & Highest & High \\
\hline Ride comfort & Bad & Medium & Good & Best \\
\hline Handling & Bad & Medium & Good & Best \\
\hline Reliability & Highest & High & Medium & High \\
\hline Dynamic & Passive & Passive & Medium & Good \\
\hline
\end{tabular}

\section{CONCLUSIONS}

In this review, the recent studies on vehicle suspension systems have been thoroughly examined. Due to its energy regeneration, structural simplicity, wide bandwidth operation, versatile and precise power control, good ride quality, and its efficiency, active electromagnetic suspensions are postulated as the future trend of car suspension designs. Stable and dynamic state performance, reduced weight and volume, unlimited integration of the electronic control system in cars, reliability, and contraction are crucial factors in rationalizing the broader use of electric transmission. Advances in this technological area require exploring the possibility of applying suspension systems usable with electromagnetic actuators to improve their efficiency without increasing costs and energy consumption. In general, the designer should consider certain requirements while designing the vehicle suspension system like independency, good camber control, good body roll control, good structural efficiency, good isolation, low weight, long life, low cost, etc. Further research on electromagnetic active suspensions should focus on two main perspectives, namely the development of electromagnetic linear drives, as well as better system management.

\section{References}

1. Cao D, Song X, Ahmadian M. Editors' perspectives: road vehicle suspension design, dynamics, and control. Vehicle System Dynamics 2011;49:3-28.

2. Ghazaly NM, Moaaz AO. The future development and analysis of vehicle active suspension system. IOSR Journal of Mechanical and Civil Engineering 2014;11:19-25.

3. Goodarzi A, Khajepour A. Vehicle suspension system technology and design. Synthesis Lectures on Advances in Automotive Technology 2017;1:i-77.

4. Dishant E, Singh P, Sharma M. Suspension systems: A review. International Research Journal of Engineering and Technology 2017;4:148-160.

5. Palangsavar N, Mamouri AR. Stability investigation of hydraulic interconnected suspension system of a vehicle with a quaternion neural network controller. Iranian Journal of Mechanical Engineering Transactions of the ISME 2019;20:129-151.

6. Zhao L, Zhou C, Yu Y. Comfort Improvement of a Novel Nonlinear Suspension for a Seat System Based on Field Measurements. Strojniski Vestnik/Journal of Mechanical Engineering 2017;63.

7. Hadi NJ, Abd Al-Hussain RK. Physical properties improvement of the diesel engine lubricant oil reinforced nanomaterials. Journal of Mechanical and Energy Engineering 2018;2:233-244.

8. Popovic V, Vasic B, Petrovic M, Mitic S. System approach to vehicle suspension system control in CAE environment. Strojniški Vestnik-Journal of Mechanical Engineering 2011;57:100-109.

9. Riduan AFM, Tamaldin N, Sudrajat A, Ahmad F. Review on active suspension system. SHS Web of Conferences, vol. 49, EDP Sciences; 2018, p. 02008.

10. Sharp R, Hassan S. The relative performance capabilities of passive, active and semi-active car suspension systems. Proceedings of the Institution of Mechanical Engineers, Part D: Transport Engineering 1986;200:219-228.

11. Inoue $H$, Yamaguchi $T$, Kondo T. Damping force generation system and vehicle suspension system constructed by including the same. Google Patents; 2010.

12. Bello MM, Babawuro AY, Fatai S. Active suspension force control with electro-hydraulic actuator dynamics. ARPN J Eng Appl Sci 2015;10:17327-17331.

13. Martins I, Esteves $M$, Da Silva FP, Verdelho P., Electromagnetic hybrid active-passive vehicle suspension system. 1999 IEEE 49th Vehicular Technology Conference (Cat. No. 99CH36363), vol. 3, IEEE; 1999, p. 2273-2277.

14. Moheyeldein MM, Abd-El-Tawwab AM, El-gwwad KA, Salem MMM. An analytical study of the performance indices of air spring suspensions over the passive suspension. Beni-Suef University Journal of Basic and Applied Sciences 2018;7:525-534.

15. Sawant SH, Tamboli DJ. Analysis and comparison of vehicle dynamic system with nonlinear parameters subjected to actual random road excitations. International Journal of Mechanical Engineering and Technology 2012;3:284-299.

16. Ahmad I, Khan A. A Comparative Analysis of Linear and Nonlinear Semi-Active Suspension System 2018.

17. Shafie AA, Bello MM, Khan RM. Active vehicle suspension control using electro hydraulic actuator on rough road terrain. Journal of Advanced Research 2015;9:15-30.

18. Dowds P, O'Dwyer A. Modelling and control of a suspension system for vehicle applications 2005.

19. Pan H, Sun W, Jing X, Gao H, Yao J. Adaptive tracking control for active suspension systems with non-ideal actuators. Journal of Sound and Vibration 2017;399:2-20. 
20. Bello MM, Babawuro AY, Fatai S. Active suspension force control with electro-hydraulic actuator dynamics. ARPN Journal of Engineering and Applied Sciences 2015;10:17327-17331.

21. [Advantages and Drawbacks of Pneumatic, Hydraulic, and Electric Linear Actuators - Barbaadachiisaa Google n.d.

\section{Biographical notes}

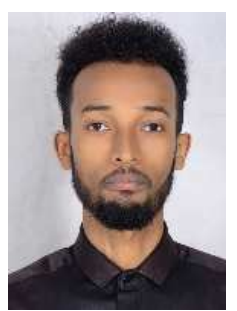

Iyasu T. Jiregna was born in Billa town, west Wollega Zone, Ethiopia in 1992. He graduated his BSc degree in Mechanical Engineering from Jimma University in 2015 and M.Sc. degree in Mechanical Design from Jimma University, 2019. Currently, he is a lecturer at Faculty of Mechanical Engineering, Jimma university. His research interests are dynamics modelling, fracture mechanics, tribology, thermomechanical effect in mechanical structures, etc.

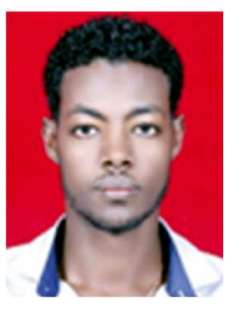

Goftila G. Sirata was born in Gorosole, West Shoa Zone, Ethiopia in 1991. He received his $\mathrm{BSc}$ degree in Mechanical Engineering from Jimma University in July 2015. He graduated with masters of science degree in Mechanical Design from Jimma University, Ethiopia in 2019. Currently, he is a PhD student at Faculty of Material Engineering, Silesian University of Technology, Poland. His scientific research interests are mechanical design system, fatigue, creep and relaxation modelling in mechanical structures. 